\title{
ANÁLISIS ESPACIAL DE LOS ACCIDENTES DE TRÁNSITO EN LA REGIÓN AMAZÓNICA, ANDINA Y COSTA DEL PACÍFICO ECUATORIANA
}

\author{
SPATIAL ANALYSIS OF TRAFFIC ACCIDENTS IN THE AMAZONIAN, \\ ANDEAN AND PACIFIC COAST REGIONS OF ECUADOR
}

\author{
ANTONIO GÓMEZ GARCÍA', GABRIELA LAHUATE ALARCÓN², YOLIS CAMPOS VILLALTA ${ }^{3}$, PABLO SUASNAVAS BERMÚDEZ ${ }^{4}$ \\ 1 Universidad Internacional SEK, Ecuador. antonio.gomez@uisek.edu.ec \\ 2 Universidad Internacional SEK, Ecuador. gabriela-pla@hotmail.com \\ 3 Universidad Internacional SEK, Ecuador. yoliks.campos@uisek.edu.ec \\ 4 Universidad Internacional SEK, Ecuador. pablo.suasnavas@uisek.edu.ec
}

RESUMEN

Los accidentes de tránsito son un problema de salud pública a nivel mundial. En Ecuador, durante el período de 2000 a 2015 se registraron un total de 338.442 accidentes de tránsito, originando 233.794 víctimas con lesión y 26.811 fallecidos. En la actualidad, son escasas las investigaciones que permitan identificar geográficamente esta problemática en la totalidad del país. Se realizó un estudio exploratorio, descriptivo y transversal con representación espacial sobre la accidentabilidad, lesividad y letalidad por accidentes de tránsito registrados en las provincias y cantones del Ecuador en 2016 a partir de fuentes oficiales de información secundaria. Los cantones con mayores tasas de letalidad ajustada (x100 siniestros por accidentes de transito) fueron; Orellana $(38,6)$ y Lago Agrio $(25,0)$ en la Región Amazónica; Tulcán $(16,0)$, Latacunga $(9,1)$, La Troncal $(7,8)$ y Montufar $(7,6)$ en la Región Andina y; Santo Domingo de los Tsachilas $(8,6)$ en la Región Costa Ecuatoriana. Los resultados de este análisis proporcionan información valiosa para la identificación de aquellos cantones provinciales del país más problemáticos y aportando evidencias para el establecimiento de programas y acciones puntuales en seguridad vial.

Palabras ClaVE: accidentes de tránsito, análisis espacial, letalidad, Ecuador.
Traffic accidents are a public health problem worldwide. In Ecuador, during the period from 2000 to 2015 a total of 338.442 traffic accidents were registered, causing 233.794 victims with injuries and 26.811 deaths. At present, there are few investigations that allow to identify this problem geographically in the whole country. Exploratory, descriptive and transverse study with spatial representation on the accidentabilidad, harmfulness and letalidad for accidents of traffic registered in the provinces and cantons of the Ecuador in 2016 from official sources of secondary information. The cantons with the highest adjusted lethality rates (x100 accidents due to traffic accidents) were; Orellana $(38,6)$ and Lago Agrio $(25,0)$ in the Amazonian Region; Tulcán $(16,0)$, Latacunga $(9,1)$, La Troncal $(7,8)$ y Montufar $(7,6)$ in the Andean Region and; Santo Domingo of the Tsachilas $(8,6)$ in the Region Ecuadorian Coast. The results of this analysis provide valuable information for the identification of those most problematic provincial cantons of the country and contributing evidences for the establishment of programs and punctual actions in road safety.

KEYWORDS: accidents, traffic, spatial analysis, lethality, Ecuador. 
INTRODUCCIÓN

Los accidentes de tránsito (AT) ocasionan anualmente 1,25 millones de personas fallecidas a nivel mundial (WHO, 2007; WHO, 2015). En países de la Región de las Américas representan la segunda causa de muerte (PAHO, 2013), constituyendo un problema prioritario en salud pública para la Organización Mundial de la Salud por el aumento de las tasas de mortalidad y los costos económicos e impactos sociales, debido principalmente al aumento de la población y del parque automotriz en las últimas décadas (Mayou \& Bryant, 2003; Ameratunga, Hijar \& Norton, 2006).

La situación sobre esta problemática en Ecuador no es muy diferente (Algora Buenafé, et al., 2017a). En el período de 2000 a 2015 se registraron un total de $338.442 \mathrm{AT}$, originando 233.794 víctimas con lesión y 26.811 fallecidos, datos estrechamente relacionados con el aumento del parque vehicular, si bien las tasas de accidentabilidad y letalidad por AT muestran un ligero descenso, la lesividad presenta una tendencia al aumento (Algora Buenafé, et al., 2017b).

No obstante, los descensos apreciados sobre la accidentabilidad y letalidad no reflejan una disminución efectiva de la problemática y enmascaran la realidad de los AT en el país (Bastos, et al., 2015; Restrepo, Medina \& Vallejo, 2016), en particular, la población joven (conductores, pasajeros y peatones) sigue siendo la más afectada (Algora Buenafé, et al., 2017a).

Los AT han sido estudiados desde múltiples enfoques (Híjar, et al., 2012; Staton, et al., 2016 ; Rissanen, Berg \& Hasselberg, 2017). Concretamente, en las últimas décadas, la literatura científica aporta valiosos métodos y conocimientos sobre la distribución de esta problemática desde un enfoque geográfico como determinante clave para analizar las relaciones existentes entre la localización y los AT (Cheng \& Washington, 2005; Montella, 2010).

Estudios realizados en otros países demuestran que los AT tienden a concentrarse en ciertas zonas geográficas, estas agrupaciones evidencian la dependencia espacial entre los accidentes y densidad poblacional, permitiendo identificar zonas o puntos críticos (puntos negros) y establecer campañas de seguridad vial, así como, la evaluación y efectividad de las políticas públicas en esta materia (Fuentes \& Hernández, 2009; Hernández, 2012).

Del mismo modo, en cuanto a los resultados de estudios ecuatorianos sobre los AT y su distribución geográfica, revelan que el 62\% de fallecimientos tuvieron lugar en áreas ubicadas fuera del perímetro urbano (zonas rurales), mientras que el $38 \%$ ocurrieron dentro del entramado urbano o en travesías (zona urbana), principalmente en las provincias con mayor densidad poblacional, en particular, en las provincias de la región amazónica por cada 100 AT se registraron 98 víctimas con lesión y $24 \mathrm{fa}-$ llecidos (Galarza, et al., 2017; Algora Buenafé, et al., 2017c).

En función a los antecedentes anteriormente expuestos y considerando que hasta el momento no existen estudios que analicen geográficamente la problemática tratada en la totalidad del país con mayor precisión, el objetivo del presente trabajo es representar espacialmente los AT registrados en las provincias y cantones del Ecuador.

Los resultados obtenidos en este estudio supondrán una importante contribución al campo de la seguridad vial, permitiendo identificar zonas geográficas de mayor accidentabilidad, lesividad y letalidad por AT para el establecimiento de programas provinciales y cantonales.

\section{MATERIALES Y MÉTODOS DISEÑO}

Se trata de un análisis de tipo exploratorio, descriptivo y transversal con representación espacial sobre la accidentabilidad, lesividad y letalidad por AT registrados en las provincias y cantones del Ecuador en el año 2016, sin incluir la región insular (Islas Galápagos).

FUENTES DE INFORMACIÓN

Los datos de la presente investigación se obtuvieron a partir de dos fuentes oficiales de información secundaria. En primer lugar, a través del Instituto Nacional de Estadística y Censos (INEC, 2010) la distribución de la población ecuatoriana por provincia y cantón a partir del último censo poblacional disponible en 2010; en segundo lugar, los datos sobre AT se obtuvieron de la Agencia Nacional de Tránsito (ANT, 2016), organismo encargado de registrar las estadísticas de accidentes a nivel nacional, así como, de regular y controlar la gestión del transporte terrestre y la seguridad vial en el país.

Atendiendo a la normativa de referencia existente, los AT se definen como todo suceso eventual o acción involuntaria, que como efecto de una o más causas y con independencia del grado de estas, ocurre en vías o lugares destinados al uso público o privado, ocasionando personas muertas, individuos con lesiones de 
diversa gravedad o naturaleza y daños materiales en vehículos, vías o infraestructura, con la participación de los usuarios de la vía, vehículo, vía y/o entorno (ANT, 2008; ANT, 2011).

ANÁLISIS ESPACIAL

En una primera fase, previa a la representación espacial, se procedió a la recopilación de información sobre los siniestros, lesionados y fallecimientos por AT clasificados por la Agencia Nacional de Tránsito, confección de una base de datos en SPSS Statistics (versión 24) y depuración de calidad de los datos. Asimismo, los datos se agruparon en unidades geográficas por (i) región ecuatoriana (Amazónica, Andina, Costa del Pacífico); (ii) provincias y, a su vez, (iii) por cantones provinciales.

Con el fin de representar espacialmente los accidentes de tránsito a nivel nacional, en una segunda fase se calcularon las tasas brutas y ajustadas:

TASAS DE ACCIDENTABILIDAD:

$T A A T_{b}=\frac{N S A T}{N P_{r}} \times 100.000 \quad T_{A A T_{a}}=\frac{N S A T}{N P_{p}} \times 100.000$

- $\mathrm{TAAT}_{\mathrm{b}}=$ Tasa de Accidentabilidad Bruta por Accidentes de Tránsito (por cada 100.000 habitantes).

- TAAT $_{a}=$ Tasa de Accidentabilidad Ajustada por Accidentes de Tránsito (por cada 100.000 habitantes).

- NSAT = Número total de Siniestros por Accidentes de Tránsito por Cantón Provincial.

- $\mathrm{NP}_{\mathrm{r}}=$ Número total de habitantes por Región.

- $\mathrm{NP}_{\mathrm{p}}=$ Número total de habitantes por Provincia.

TASAS DE LESIVIDAD:

$T L S A T_{b}=\frac{N L A T}{N A T_{r}} \times 1.000 \quad T L S A T_{a}=\frac{N L A T}{N A T_{p}} \times 100$

- $\operatorname{TLSAT}_{\mathrm{b}}=$ Tasa de Lesividad Bruta por Accidentes de Tránsito (por cada 1.000 siniestros).

- TLSAT $_{a}=$ Tasa de Lesividad Ajustada por Accidentes de Tránsito (por cada 100 siniestros).

- NLAT = Número total de Lesionados por Accidentes de Tránsito por Cantón Provincial.
- $\mathrm{NAT}_{\mathrm{r}}=$ Número total de Siniestros por Región.

- $\mathrm{NAT}_{\mathrm{p}}=$ Número total de Siniestros por Provincia.

TASAS DE LETALIDAD:

$T L E A T_{b}=\frac{N F A T}{N A T_{r}} \times 1.000 \quad T L E A T_{a}=\frac{N F A T}{N A T_{p}} \times 100$

- $\operatorname{TLEAT}_{\mathrm{b}}=$ Tasa de Letalidad Bruta por Accidentes de Tránsito (por cada 1.000 siniestros).

- $\operatorname{TLEAT}_{\mathrm{a}}=$ Tasa de Letalidad Ajustada por Accidentes de Tránsito (por cada 100 siniestros).

- NFAT= Número total de Fallecidos por Accidentes de Tránsito por Cantón Provincial.

- $\mathrm{NAT}_{\mathrm{r}}=$ Número total de Siniestros por Región.

- $\mathrm{NAT}_{\mathrm{p}}=$ Número total de Siniestros por Provincia.

A través de la revisión de la literatura científica, se justifica el empleo de estos indicadores puesto que han sido empleados en el contexto ecuatoriano (Algora Buenafé, et al., 2017a; Algora Buenafé, et al., 2017b) y a nivel internacional (Kumar, et al., 2012; McWade, et al., 2017; Qin, et al., 2017), permitiendo establecer diferencias poblaciones y geográficas de los AT y orientan a la toma de decisiones públicas en seguridad vial. Asimismo, se estudió la correlación entre la población de cada uno de los cantones por provincia y sus respectivas tasas ajustadas de lesividad y letalidad por AT mediante el empleo del Coeficiente de Correlación de Pearson $(\mathrm{p}<0,05 ; \mathrm{p}<0,01 ; \mathrm{p}<0,001)$.

Se empleó la aplicación Power View de Microsoft Excel para la visualización y presentación en mapas de las principales provincias con mayor tasa ajustada de accidentabilidad, lesividad y letalidad.

\section{RESULTADOS}

La población en Ecuador se sitúa en 14.383.067 habitantes, según el último censo poblacional de 2010 sin incluir las Islas Galápagos. Los siniestros por AT registrados en 2016 fueron 30.263 , los cuales originaron 19.027 víctimas con lesión y 1.450 personas fallecidas por esta causa. En el Figura 1 ( $A-B-C)$ se observa que los siniestros, víctimas con lesión y fallecidos por AT se concentran principalmente en la Región 
A. Siniestros por AT $(n=30.263)$

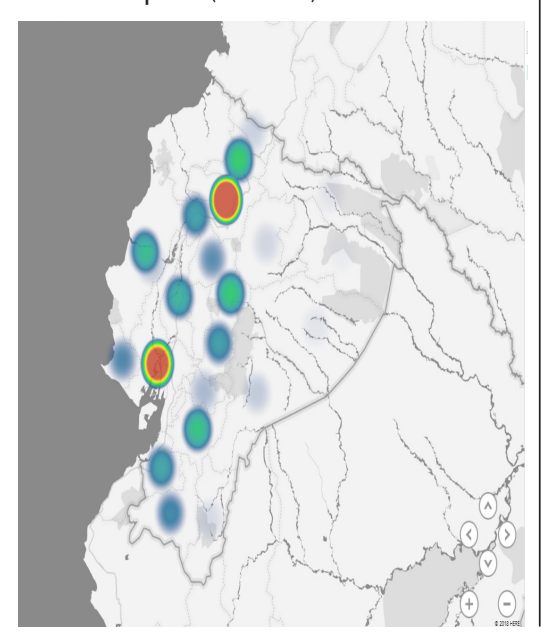

B. Lesiones por AT $(\mathrm{n}=20.509)$.

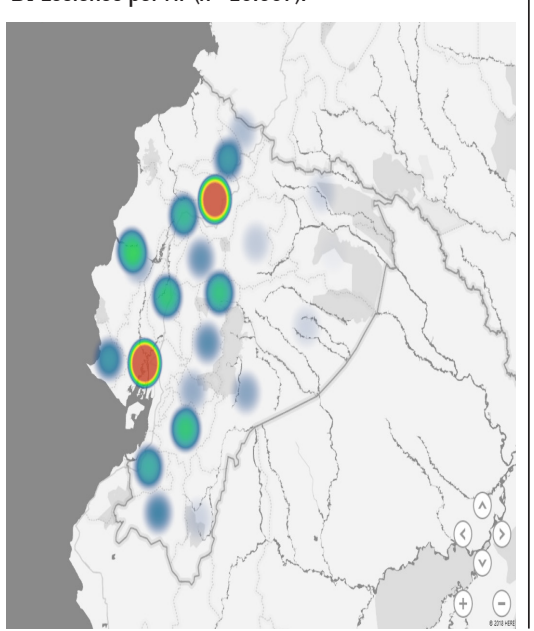

C. Fallecidos por AT ( $\mathrm{n}=1.450)$.

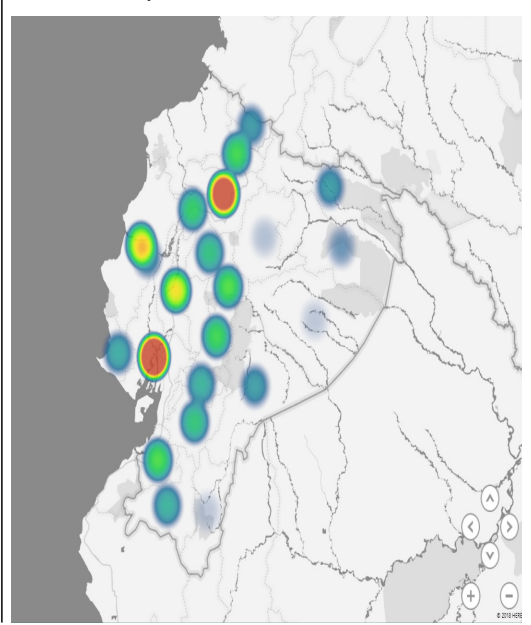

Figura 1 (A-B-C). Distribución geográfica de los siniestros, víctimas con lesión y fallecidos por AT.

Andina (centro-norte) y Región Costa del Pacífico (sur-este). A nivel nacional, por cada 1.000 siniestros 629 personas sufrieron lesiones y por cada 100 siniestros 5 personas fallecieron por AT, siendo la tasa de accidentabilidad de 210,4 por cada 100.000 habitantes.

A continuación, se presentan los resultados de los siniestros, lesionados, fallecimientos, tasas por AT y su representación espacial según provincias y cantones del Ecuador.

\section{REGIÓN AMAZÓNICA ECUATORIANA}

En la Región Amazónica, con una población de 739.814 habitantes, se registraron un total de 556 siniestros, 547 víctimas con lesión y 126 personas fallecidas en AT. Por cada 1.000 AT, 984 personas sufrieron lesiones y 227 fallecieron. La tasa de accidentabilidad en esta región se sitúa en 75 por cada 100.000 habitantes.

Morona Santiago y Sucumbíos son las provincias de la región con las tasas de letalidad (Bruta) por AT más elevadas, 64,7 fallecidos por cada 1.000 siniestros respectivamente, ver Tabla 1 . Asimismo, se observa que Morona Santiago presenta la mayor tasa de lesividad bruta (336,3 x1.000 siniestros) y accidentabilidad (21,1 x100.000 habitantes), seguido de la provincia de Napo (174,5 x1.000 siniestros; 15,5 x100.000 habitantes).

En cuanto a las tasas de letalidad ajustadas por cantones provinciales, se observa que Orellana ( $\mathrm{n}=17 ; 13,5 \%$ fallecidos) por cada 100 siniestros causaron la muerte a 38,6 personas y 25,0 en Lago Agrio ( $n=18 ; 14,3 \%$ fallecidos). Lago Agrio ( $\mathrm{n}=66 ; 12,1 \%$ lesionados) y Pastaza ( $\mathrm{n}=53 ; 9,7 \%$ lesionados) son los cantones con mayor tasa de lesividad; 91,7 y 77,9 víctimas con lesión cada 100 siniestros por AT.
Finalmente, los cantones provinciales de la Región Amazónica que representan mayores tasas ajustadas de accidentabilidad son Zamora $(n=59 ; 10,6 \%$ sinestros) con 64,6 siniestros por cada 100.000 habitantes, Pastaza $(n=49 ; 8,8 \%$ sinestros) con 58,4 y con 51,1 por cada 100.000 habitantes el cantón de Tena ( $\mathrm{n}=53 ; 9,5 \%$ sinestros).

Se observa una relación estadísticamente significativa entre número de habitantes y la tasa de lesividad en los cantones provinciales de Pastaza $(\mathrm{p}<0,01)$.

Igualmente, se observa una asociación respecto a la tasa de letalidad en Napo, Orellana, Pastaza y Sucumbíos $(\mathrm{p}<0,01)$. No encontrandose asociación estadísticamentes significativa para el resto de cantones provinciales de la Región Amazónica Ecuatorina.

\section{REGIÓN ANDINA ECUATORIANA}

Se registraron un total de 17.333 siniestros, 8.843 víctimas con lesión y 421 personas fallecidas en AT. La tasa de accidentabilidad se sitúa en 285 por cada 100.000 habitantes en la región (6.081.342 habitantes), por cada $1.000 \mathrm{AT}, 510$ personas sufrieron lesiones y 24 fallecieron. La provincia de Pichicha presenta la mayor tasa de accidentabilidad bruta por AT (177,2 x100.000 habitantes), lesividad (316,4 x1.000 siniestros) y letalidad (19,1 x1.000 siniestros) de la Región Andina Ecuatoriana, ver Tabla 2. En un segundo orden y no descartando su importancia, destacan las provincias de Imbabura $(25,3)$, Tungurahua $(23,7)$ y Azuay $(21,5)$ por su tasas de accidentabilidad bruta; Azuay $(49,0)$ y Tungurahua $(45,1)$ por sus tasas de lesividad bruta y; Tungurahua $(4,8)$, Imbabura $(4,5)$ y Chimborazo $(4,4)$ por sus tasas de letalidad bruta en la región. 
TABLA 2. TASAS DE LOS A.T. SEGÚN PROVINCIAS Y CANTONES EN LA REGIÓN ANDINA ECUATORIANA.

\begin{tabular}{|c|c|c|c|c|c|c|c|c|c|}
\hline & SINIESTROS & LESIONADOS & FALLECIDOS & \multicolumn{2}{|c|}{ ACCIDENTABILIDAD } & \multicolumn{2}{|c|}{ LESIVIDAD } & \multicolumn{2}{|c|}{ LETALIDAD } \\
\hline AZUAY & $1.310(7,6)$ & $849(9,6)$ & $61(7,2)$ & 21,5 & 184 & 49 & $64,8^{*}$ & 3,5 & $4,7^{* *}$ \\
\hline Camilo P.E. & $26(2,0)$ & $16(1,9)$ & $3(4,9)$ & 0,4 & 3,7 & 0,9 & 1,2 & 0,2 & 0,2 \\
\hline Chordeleg & $1(0,1)$ & - & $7(11,5)$ & 0 & 0,1 & 0 & 0 & 0,4 & 0,5 \\
\hline & $N(\% N)$ & N (\%N) & N (\%N) & TAATB & TAATA & TLSATB & TLSATA & TLEATB & TLEATA \\
\hline Cuenca & $1.076(82,1)$ & $756(89,0)$ & $30(49,2)$ & 17,7 & 151,1 & 43,6 & 57,7 & 1,7 & 2,3 \\
\hline El Pan & - & - & - & - & - & - & - & - & - \\
\hline Girón & $36(2,7)$ & $6(0,7)$ & $3(4,9)$ & 0,6 & 5,1 & 0,3 & 0,5 & 0,2 & 0,2 \\
\hline Guachapala & $1(0,1)$ & $5(0,6)$ & - & 0 & 0,1 & 0,3 & 0,4 & - & - \\
\hline Gualaceo & $56(4,3)$ & $19(2,2)$ & $5(8,2)$ & 0,9 & 7,9 & 1,1 & 1,5 & 0,3 & 0,4 \\
\hline Nabón & $13(1,0)$ & - & - & 0,2 & 1,8 & - & - & - & - \\
\hline Oña & $4(0,3)$ & - & - & 0,1 & 0,6 & - & - & - & - \\
\hline Paute & $50(3,8)$ & $24(2,8)$ & $8(13,1)$ & 0,8 & 7 & 1,4 & 1,8 & 0,5 & 0,6 \\
\hline Pucará & $5(0,4)$ & $3(0,4)$ & $1(1,6)$ & 0,1 & 0,7 & 0,2 & 0,2 & 0,1 & 0,1 \\
\hline San Fernando & - & - & - & - & - & - & - & - & - \\
\hline Santa Isabel & $31(2,4)$ & $6(0,7)$ & - & 0,5 & 4,4 & 0,3 & 0,5 & - & - \\
\hline Sevilla Oro & $1(0,1)$ & - & - & 0 & 0,1 & - & - & - & - \\
\hline Sisgsig & $10(0,8)$ & $14(1,6)$ & $4(6,6)$ & 0,2 & 1,4 & 0,8 & 1,1 & 0,2 & 0,3 \\
\hline BoLÍVAR & $193(1,1)$ & $188(2,1)$ & $33(3,9)$ & 3,2 & 10,5 & 10,8 & 9,7 & 1,9 & 17,1 \\
\hline Caluma & $5(2,6)$ & $2(1,1)$ & $2(6,1)$ & 0,1 & 2,7 & 0,1 & 1 & 0,1 & 1 \\
\hline Chillanes & $7(3,6)$ & $10(5,3)$ & $1(3,0)$ & 0,1 & 3,8 & 0,6 & 5,2 & 0,1 & 0,5 \\
\hline Chimbo & $16(8,3)$ & $9(4,8)$ & $2(6,1)$ & 0,3 & 8,7 & 0,5 & 4,7 & 0,1 & 1 \\
\hline Echeandía & $9(4,7)$ & $11(5,9)$ & $4(12,1)$ & 0,1 & 4,9 & 0,6 & 5,7 & 0,2 & 2,1 \\
\hline Guaranda & $125(64,8)$ & $122(64,9)$ & $13(39,4)$ & 2,1 & 68,1 & 7 & 63,2 & 0,8 & 6,7 \\
\hline Las Naves & $2(1,0)$ & - & $5(15,2)$ & 0 & 1,1 & 0 & 0 & 0,3 & 2,6 \\
\hline San Miguel & $29(15,0)$ & $34(18,1)$ & $6(18,2)$ & 0,5 & 15,8 & 2 & 17,6 & 0,3 & 3,1 \\
\hline CAÑAR & $218(1,3)$ & $168(1,9)$ & $47(5,5)$ & 3,6 & 96,8 & 9,7 & 77,1 & 2,7 & 21,6 \\
\hline Azogues & $61(28,0)$ & $42(25,0)$ & $7(14,9)$ & 1 & 27,1 & 2,4 & 19,3 & 0,4 & 3,2 \\
\hline Biblián & $25(11,5)$ & $8(4,8)$ & $3(6,4)$ & 0,4 & 11,1 & 0,5 & 3,7 & 0,2 & 1,4 \\
\hline Cañar & $50(22,9)$ & $50(29,8)$ & $14(29,8)$ & 0,8 & 22,2 & 2,9 & 22,9 & 0,8 & 6,4 \\
\hline Déleg & $3(1,4)$ & $2(1,2)$ & $2(4,3)$ & 0 & 1,3 & 0,1 & 0,9 & 0,1 & 0,9 \\
\hline El Tambo & $14(6,4)$ & $13(7,7)$ & $3(6,4)$ & 0,2 & 6,2 & 0,8 & 6 & 0,2 & 1,4 \\
\hline La Troncal & $60(27,5)$ & $51(30,4)$ & $17(36,2)$ & 1 & 26,6 & 2,9 & 23,4 & 1 & 7,8 \\
\hline Suscal & $5(2,3)$ & $2(1,2)$ & $1(2,1)$ & 0,1 & 2,2 & 0,1 & 0,9 & 0,1 & 0,5 \\
\hline CARCHI & $131(0,8)$ & $126(1,4)$ & $34(4,0)$ & 2,2 & 79,6 & 7,3 & $96,2^{* *}$ & 2 & $26,0^{*}$ \\
\hline Bolívar & $8(6,1)$ & $9(7,1)$ & $3(8,8)$ & 0,1 & 4,9 & 0,5 & 6,9 & 0,2 & 2,3 \\
\hline Espejo & $2(1,5)$ & $5(4,0)$ & - & 0 & 1,2 & 0,3 & 3,8 & - & - \\
\hline Mira & $3(2,3)$ & $9(7,1)$ & - & 0 & 1,8 & 0,5 & 6,9 & - & - \\
\hline Montufar & $24(18,3)$ & $18(14,3)$ & $10(29,4)$ & 0,4 & 14,6 & 1 & 13,7 & 0,6 & 7,6 \\
\hline San Pedro H. & $2(1,5)$ & $1(0,8)$ & - & 0 & 1,2 & 0,1 & 0,8 & - & - \\
\hline Tulcán & $92(70,2)$ & $84(66,7)$ & $21(61,8)$ & 1,5 & 55,9 & 4,8 & 64,1 & 1,2 & 16 \\
\hline CHIMBORAZO & $743(4,3)$ & $263(3,0)$ & $77(9,1)$ & 12,2 & 162 & 15,2 & $35,4^{* *}$ & 4,4 & $10,4^{*}$ \\
\hline Alausí & $19(2,6)$ & $38(14,4)$ & $8(10,4)$ & 0,3 & 4,1 & 2,2 & 5,1 & 0,5 & 1,1 \\
\hline Chambo & $8(1,1)$ & $2(0,8)$ & $4(5,2)$ & 0,1 & 1,7 & 0,1 & 0,3 & 0,2 & 0,5 \\
\hline Chunchi & $8(1,1)$ & $6(2,3)$ & $7(9,1)$ & 0,1 & 1,7 & 0,3 & 0,8 & 0,4 & 0,9 \\
\hline Colta & $52(7,0)$ & $33(12,5)$ & $8(10,4)$ & 0,9 & 11,3 & 1,9 & 4,4 & 0,5 & 1,1 \\
\hline Cumandá & $4(0,5)$ & $2(0,8)$ & - & 0,1 & 0,9 & 0,1 & 0,3 & - & - \\
\hline Guamote & $11(1,5)$ & $1(0,4)$ & $1(1,3)$ & 0,2 & 2,4 & 0,1 & 0,1 & 0,1 & 0,1 \\
\hline Guano & $56(7,5)$ & $29(11,0)$ & $9(11,7)$ & 0,9 & 12,2 & 1,7 & 3,9 & 0,5 & 1,2 \\
\hline Pallatanga & $17(2,3)$ & $12(4,6)$ & $7(9,1)$ & 0,3 & 3,7 & 0,7 & 1,6 & 0,4 & 0,9 \\
\hline Penipe & $3(0,4)$ & $1(0,4)$ & - & 0 & 0,7 & 0,1 & 0,1 & - & - \\
\hline Riobamba & $565(76,0)$ & $139(52,9)$ & $33(42,9)$ & 9,3 & 123,2 & 8 & 18,7 & 1,9 & 4,4 \\
\hline СОТОРАХI & $427(2,5)$ & $249(2,8)$ & $58(6,8)$ & 7 & 104,3 & 14,4 & 58,3 & 3,3 & $0,5^{* *}$ \\
\hline La Maná & $14(3,3)$ & $26(10,4)$ & $2(3,4)$ & 0,2 & 0,5 & 1,5 & 6,1 & 0,1 & 0,5 \\
\hline Latacunga & $318(74,5)$ & $170(68,3)$ & $39(67,2)$ & 5,2 & 12,3 & 9,8 & 1,6 & 2,3 & 9,1 \\
\hline Pangua & - & - & - & - & - & - & - & - & - \\
\hline Pujilí & $24(5,6)$ & $20(8,0)$ & $6(10,3)$ & 0,4 & 0,9 & 1,2 & 0,2 & 0,3 & 1,4 \\
\hline Salcedo & $61(14,3)$ & $29(11,6)$ & $8(13,8)$ & 1 & 2,4 & 1,7 & 0,3 & 0,5 & 1,9 \\
\hline Saquisilí & $6(1,4)$ & $3(1,2)$ & $2(3,4)$ & 0,1 & 0,2 & 0,2 & 0 & 0,1 & 0,5 \\
\hline Sigchos & $4(0,9)$ & $1(0,4)$ & $1(1,7)$ & 0,1 & 0,2 & 0,1 & 0 & 0,1 & 0,2 \\
\hline IMBABURA & $1.536(8,9)$ & $434(4,9)$ & $78(9,2)$ & 25,3 & 385,7 & 25 & $28,3^{*}$ & 4,5 & 0,7 \\
\hline Antonio Ante & $66(4,3)$ & $38(8,8)$ & $15(19,2)$ & 1,1 & 2,6 & 2,2 & 0,4 & 0,9 & 1 \\
\hline Cotacachi & $38(2,5)$ & $43(9,9)$ & $6(7,7)$ & 0,6 & 1,5 & 2,5 & 0,4 & 0,3 & 0,4 \\
\hline Ibarra & $1.286(83,7)$ & $264(60,8)$ & $35(44,9)$ & 21,1 & 49,9 & 15,2 & 2,4 & 2 & 2,3 \\
\hline
\end{tabular}




\begin{tabular}{|c|c|c|c|c|c|c|c|c|c|}
\hline & \multirow{2}{*}{$\begin{array}{r}\text { SINIESTROS } \\
\mathrm{N}(\% \mathrm{~N})\end{array}$} & \multirow{2}{*}{$\begin{array}{r}\text { LESIONADOS } \\
N(\% N)\end{array}$} & \multirow{2}{*}{$\begin{array}{r}\text { FALLECIDOS } \\
N(\% \mathrm{~N})\end{array}$} & \multicolumn{2}{|c|}{ ACCIDENTABILIDAD } & \multicolumn{2}{|c|}{ LESIVIDAD } & \multicolumn{2}{|c|}{ LETALIDAD } \\
\hline & & & & ТАATB & TAATA & TLSATB & TLSATA & TLEATB & TLEATA \\
\hline Otavalo & $131(8,5)$ & $81(18,7)$ & $15(19,2)$ & 2,2 & 5,1 & 4,7 & 0,8 & 0,9 & 1 \\
\hline Pimampiro & $4(0,3)$ & - & $5(6,4)$ & 0,1 & 0,2 & - & - & 0,3 & 0,3 \\
\hline San Miguel U. & $11(0,7)$ & $8(1,8)$ & $2(2,6)$ & 0,2 & 0,4 & 0,5 & 0,1 & 0,1 & 0,1 \\
\hline LOJA & $559(3,2)$ & $300(3,4)$ & $47(5,5)$ & 9,2 & 124,5 & 17,3 & $53,7^{* *}$ & 2,7 & 8,4 \\
\hline Calvas & $16(2,9)$ & $22(7,3)$ & $2(4,3)$ & 0,3 & 3,6 & 1,3 & 3,9 & 0,1 & 0,4 \\
\hline Catamayo & $23(4,1)$ & $35(11,7)$ & $11(23,4)$ & 0,4 & 5,1 & 2 & 6,3 & 0,6 & 2 \\
\hline Celica & $4(0,7)$ & $6(2,0)$ & - & 0,1 & 0,9 & 0,3 & 1,1 & - & - \\
\hline Chaguarpamba & $2(0,4)$ & - & - & 0 & 0,4 & 0 & 0 & - & - \\
\hline Espíndola & $1(0,2)$ & $2(0,7)$ & $1(2,1)$ & 0 & 0,2 & 0,1 & 0,4 & 0,1 & 0,2 \\
\hline Gonzanamá & $2(0,4)$ & $6(2,0)$ & $2(4,3)$ & 0 & 0,4 & 0,3 & 1,1 & 0,1 & 0,4 \\
\hline Loja & $478(85,5)$ & $192(64,0)$ & $23(48,9)$ & 7,9 & 106,5 & 11,1 & 34,3 & 1,3 & 4,1 \\
\hline Macará & $5(0,9)$ & $4(1,3)$ & - & 0,1 & 1,1 & 0,2 & 0,7 & - & - \\
\hline Olmedo & $1(0,2)$ & $3(1,0)$ & - & 0 & 0,2 & 0,2 & 0,5 & - & - \\
\hline Paltas & $5(0,9)$ & $8(2,7)$ & $1(2,1)$ & 0,1 & 1,1 & 0,5 & 1,4 & 0,1 & 0,2 \\
\hline Pindal & $2(0,4)$ & $2(0,7)$ & - & 0 & 0,4 & 0,1 & 0,4 & - & - \\
\hline Puyango & $2(0,4)$ & $4(1,3)$ & - & 0 & 0,4 & 0,2 & 0,7 & - & - \\
\hline Quingala & - & - & - & - & - & - & - & - & - \\
\hline Saraguro & $11(2,0)$ & $11(3,7)$ & $4(8,5)$ & 0,2 & 2,5 & 0,6 & 2 & 0,2 & 0,7 \\
\hline Sozoranga & - & - & - & - & - & - & - & - & - \\
\hline Zapotillo & $7(1,3)$ & $5(1,7)$ & $3(6,4)$ & 0,1 & 1,6 & 0,3 & 0,9 & 0,2 & 0,5 \\
\hline PICHINCHA & $10.777(62,2)$ & $5.484(62,0)$ & $331(38,9)$ & 177,2 & 418,3 & 316,4 & $50,9 * * *$ & 19,1 & $3,1^{* *}$ \\
\hline Cayambe & $75(0,7)$ & $61(1,1)$ & $19(5,7)$ & 1,2 & 2,9 & 3,5 & 0,6 & 1,1 & 0,2 \\
\hline Mejía & $280(2,6)$ & $154(2,8)$ & $61(18,4)$ & 4,6 & 10,9 & 8,9 & 1,4 & 3,5 & 0,6 \\
\hline Pedro Moncayo & $25(0,2)$ & $12(0,2)$ & $7(2,1)$ & 0,4 & 1 & 0,7 & 0,1 & 0,4 & 0,1 \\
\hline Pedro V.M. & $5(0,0)$ & $8(0,1)$ & $3(0,9)$ & 0,1 & 0,2 & 0,5 & 0,1 & 0,2 & 0 \\
\hline Puerto Quito & $8(0,1)$ & $3(0,1)$ & $1(0,3)$ & 0,1 & 0,3 & 0,2 & 0 & 0,1 & 0 \\
\hline Quito & $10.256(95,2)$ & $5.184(94,5)$ & $233(70,4)$ & 168,6 & 398,1 & 299,1 & 48,1 & 13,4 & 2,2 \\
\hline Rumiñahui & $97(0,9)$ & $45(0,8)$ & $3(0,9)$ & 1,6 & 3,8 & 2,6 & 0,4 & 0,2 & 0 \\
\hline San M. Bancos & $31(0,3)$ & $17(0,3)$ & $4(1,2)$ & 0,5 & 1,2 & 1 & 0,2 & 0,2 & 0 \\
\hline TUNGURAHUA & $1.439(8,3)$ & $782(8,8)$ & $84(9,9)$ & 23,7 & 285,2 & 45,1 & $54,4^{* *}$ & 4,8 & $5,8^{* *}$ \\
\hline Ambato & $1.233(85,7)$ & $620(79,3)$ & $62(73,8)$ & 20,3 & 244,4 & 35,8 & 43,1 & 3,6 & 4,3 \\
\hline Baños & $24(1,7)$ & $34(4,3)$ & $6(7,1)$ & 0,4 & 4,8 & 2 & 2,4 & 0,3 & 0,4 \\
\hline Cevallos & $3(0,2)$ & $1(0,1)$ & - & 0 & 0,6 & 0,1 & 0,1 & - & - \\
\hline Mocha & $8(0,6)$ & $5(0,6)$ & - & 0,1 & 1,6 & 0,3 & 0,3 & - & - \\
\hline Patate & $14(1,0)$ & $14(1,8)$ & $1(1,2)$ & 0,2 & 2,8 & 0,8 & 1 & 0,1 & 0,1 \\
\hline Quero & $27(1,9)$ & $13(1,7)$ & $2(2,4)$ & 0,4 & 5,4 & 0,8 & 0,9 & 0,1 & 0,1 \\
\hline San Pedro P. & $89(6,2)$ & $64(8,2)$ & $8(9,5)$ & 1,5 & 17,6 & 3,7 & 4,4 & 0,5 & 0,6 \\
\hline Santiago P. & $25(1,7)$ & $12(1,5)$ & $3(3,6)$ & 0,4 & 5 & 0,7 & 0,8 & 0,2 & 0,2 \\
\hline Tisaleo & $16(1,1)$ & $19(2,4)$ & $2(2,4)$ & 0,3 & 3,2 & 1,1 & 1,3 & 0,1 & 0,1 \\
\hline
\end{tabular}

TAATb = Tasa de Accidentabilidad Bruta por Accidentes de Tránsito (por cada 100.000 habitantes). TAATa = Tasa de Accidentabilidad Ajustada por Accidentes de Tránsito (por cada 100.000 habitantes). TLSATb = Tasa de Lesividad Bruta por Accidentes de Tránsito (por cada 1.000 siniestros). TLSATa = Tasa de Lesividad Ajustada por Accidentes de Tránsito (por cada 100 siniestros). TLEATb = Tasa de Letalidad Bruta por Accidentes de Tránsito (por cada 1.000 siniestros). TLEATa = Tasa de Letalidad Ajustada por Accidentes de Tránsito (por cada 100 siniestros). Coeficiente de Correlación de Pearson ( $\left.{ }^{*} \mathrm{p}<0,05 ;{ }^{* *} \mathrm{p}<0,01 ;{ }^{* * *} \mathrm{p}<0,001\right)$.

Respecto a las tasas ajustadas de accidentabilidad por cantón provincial, por orden de importancia destaca Quito con 398,1 (Pichincha), Ambato con 244,4 (Tungurahua), Cuenca con 151,1 (Azuay), Riobamba con 123,2 (Chimborazo), Loja con 106,5 (Loja), Guaranda con 68,1 (Bolívar), Tulcán con 55,9 (Carchi), Ibarra con 49,9 (Imbabura) y con 27,1 Azogues (Cañar). Tulcán $(64,1)$, Guaranda $(63,2)$ y Cuenca $(57,7)$ son los cantones provinciales con mayores tasas de lesividad ajustada y Tulcán (16,0), Latacunga $(9,1)$, La Troncal $(7,8)$, Montufar $(7,6)$, Guaranda $(6,7)$, Cañar $(6,4)$ y Loja $(4,1)$ como los cantones con mayores tasas de letalidad ajustada.

Se observa una relación estadísticamente significativa entre número de habitantes y la tasa de lesividad ajustada en la mayoría de las provincias, excepto en Bolívar, Cañar y Cotopaxi, asimismo, en la tasa de letalidad ajustada, excepto en Bolívar, Cañar, Imbabura y Loja.

REGIÓN COSTA ECUATORIANA

La Costa del Pacífico es la región más poblada del país (7.561.911 habitantes) y registra el mayor número de casos de víctimas con lesión $(\mathrm{n}=9.637 ; 51 \%)$ y fallecidos por AT $(\mathrm{n}=903 ; 62 \%)$ $y$, la segunda en cuanto a siniestros $(n=12.374$; 41\%). La tasa de accidentabilidad se sitúa en 164 por cada 100.000 habitantes, por cada $1.000 \mathrm{AT}$, 779 personas sufrieron lesiones y 73 fallecieron.

Las provincias de la región con las tasas de letalidad bruta por AT más elevadas son; 
TABLA 3. TASAS DE LOS A.T. SEGÚN PROVINCIAS Y CANTONES EN LA REGIÓN COSTA ECUATORIANA

\begin{tabular}{|c|c|c|c|c|c|c|c|c|c|}
\hline \multirow{2}{*}{\multicolumn{2}{|c|}{$n(\% n)$}} & \multirow{2}{*}{$\begin{array}{r}\text { Lesionados } \\
n(\% n)\end{array}$} & \multirow{2}{*}{$\begin{array}{r}\text { Fallecidos } \\
n(\% n)\end{array}$} & \multicolumn{2}{|c|}{ Accidentabilidad } & \multicolumn{2}{|c|}{ Lesividad } & \multicolumn{2}{|c|}{ Letalidad } \\
\hline & & & & TAAT $_{\mathrm{b}}$ & TAAT $_{a}$ & TLSAT $_{b}$ & TLSAT $_{a}$ & TLEAT $_{b}$ & TLEAT $_{a}$ \\
\hline EL ORO & $828(6,7)$ & $584(6,1)$ & $82(9,1)$ & 10,9 & 137,8 & 47,2 & 70,5 & 6,6 & $9,9^{*}$ \\
\hline Arenilla & $68(8,2)$ & $63(10,8)$ & $10(12,2)$ & 0,9 & 11,3 & 5,4 & 7,6 & 0,9 & 1,2 \\
\hline Atahualpa & $5(0,6)$ & - & - & 0,1 & 0,8 & - & - & - & - \\
\hline Balsas & $13(1,6)$ & $6(1,0)$ & - & 0,2 & 2,2 & 0,5 & 0,7 & - & - \\
\hline Chilla & $1(0,1)$ & - & - & 0,0 & 0,2 & - & - & - & - \\
\hline El Guabo & $120(14,5)$ & $86(14,7)$ & $17(20,7)$ & 1,7 & 20,0 & 7,4 & 10,4 & 1,5 & 2,1 \\
\hline Huaquillas & $32(3,9)$ & $27(4,6)$ & $2(2,4)$ & 0,4 & 5,3 & 2,3 & 3,3 & 0,2 & 0,2 \\
\hline La Lajas & $5(0,6)$ & $2(0,3)$ & - & 0,1 & 0,8 & 0,2 & 0,2 & 0,0 & 0,0 \\
\hline Machala & $319(38,5)$ & $206(35,3)$ & $28(34,1)$ & 4,4 & 53,1 & 17,8 & 24,9 & 2,4 & 3,4 \\
\hline Marcabelí & $3(0,4)$ & $4(0,7)$ & - & 0,0 & 0,5 & 0,3 & 0,5 & - & - \\
\hline Pasaje & $108(13,0)$ & $88(15,1)$ & $11(13,4)$ & 1,5 & 18,0 & 7,6 & 10,6 & 0,9 & 1,3 \\
\hline Piñas & $72(8,7)$ & $38(6,5)$ & $8(9,8)$ & 1,0 & 12,0 & 3,3 & 4,6 & 0,7 & 1,0 \\
\hline Portovelo & $15(1,8)$ & $13(2,2)$ & - & 0,2 & 2,5 & 1,1 & 1,6 & - & - \\
\hline Santa Rosa & $59(7,1)$ & $48(8,2)$ & $4(4,9)$ & 0,8 & 9,8 & 4,1 & 5,8 & 0,3 & 0,5 \\
\hline Zaruma & $8(1,0)$ & $3(0,5)$ & $2(2,4)$ & 0,1 & 1,3 & 0,3 & 0,4 & 0,2 & 0,2 \\
\hline ESMERALDAS & $293(2,4)$ & $292(3,0)$ & $44(4,9)$ & 3,9 & 6,0 & 23,6 & 10,0 & 3,6 & $15,0^{* *}$ \\
\hline Atacames & $23(7,8)$ & $19(6,5)$ & $2(4,5)$ & 0,3 & 4,7 & 1,6 & 6,5 & 0,2 & 0,7 \\
\hline Eloy Alfaro & $8(2,7)$ & $8(2,7)$ & $2(4,5)$ & 0,1 & 1,6 & 0,7 & 2,7 & 0,2 & 0,7 \\
\hline Esmeraldas & $155(52,9)$ & $121(41,4)$ & $20(45,5)$ & 2,2 & 31,6 & 10,4 & 41,3 & 1,7 & 6,8 \\
\hline Muisne & $4(1,4)$ & $1(0,3)$ & $3(6,8)$ & 0,1 & 0,8 & 0,1 & 0,3 & 0,3 & 1,0 \\
\hline Quinindé & $86(29,4)$ & $130(44,5)$ & $13(29,5)$ & 1,2 & 17,5 & 11,2 & 44,4 & 1,1 & 4,4 \\
\hline Río Verde & $7(2,4)$ & $6(2,1)$ & $1(2,3)$ & 0,1 & 1,4 & 0,5 & 2,0 & 0,1 & 0,3 \\
\hline San Lorenzo & $10(3,4)$ & $7(2,4)$ & $3(6,8)$ & 0,1 & 2,0 & 0,6 & 2,4 & 0,3 & 1,0 \\
\hline GUAYAS & $7.899(63,8)$ & $6.630(68,8)$ & $458(50,7)$ & 104,5 & 216,7 & 535,8 & 83,9 *** & 37,0 & $5,8^{* *}$ \\
\hline Alfredo B.M. & $38(0,5)$ & $32(0,5)$ & $12(2,6)$ & 0,5 & 1,0 & 2,8 & 0,4 & 1,0 & 0,2 \\
\hline Balao & $34(0,4)$ & $29(0,4)$ & $7(1,5)$ & 0,5 & 0,9 & 2,5 & 0,4 & 0,6 & 0,1 \\
\hline Balzar & $76(1,0)$ & $52(0,8)$ & $15(3,3)$ & 1,1 & 2,1 & 4,5 & 0,7 & 1,3 & 0,2 \\
\hline Colimes & $21(0,3)$ & $12(0,2)$ & $7(1,5)$ & 0,3 & 0,6 & 1,0 & 0,2 & 0,6 & 0,1 \\
\hline Coronel M.M. & $12(0,2)$ & $5(0,1)$ & $2(0,4)$ & 0,2 & 0,3 & 0,4 & 0,1 & 0,2 & 0,0 \\
\hline Daule & $297(3,8)$ & $231(3,5)$ & $24(5,2)$ & 4,1 & 8,1 & 19,9 & 2,9 & 2,1 & 0,3 \\
\hline El Empalme & $103(1,3)$ & $89(1,3)$ & $13(2,8)$ & 1,4 & 2,8 & 7,7 & 1,1 & 1,1 & 0,2 \\
\hline El Triunfo & $100(1,3)$ & $80(1,2)$ & $12(2,6)$ & 1,4 & 2,7 & 6,9 & 1,0 & 1,0 & 0,2 \\
\hline Eloy Alfaro & $655(8,3)$ & $489(7,4)$ & $27(5,9)$ & 9,1 & 18,0 & 42,2 & 6,2 & 2,3 & 0,3 \\
\hline General A.E. & $35(0,4)$ & $26(0,4)$ & $5(1,1)$ & 0,5 & 1,0 & 2,2 & 0,3 & 0,4 & 0,1 \\
\hline Guayaquil & $4.809(60,9)$ & $4.153(62,6)$ & $173(37,8)$ & 66,8 & 131,9 & 358,6 & 52,6 & 14,9 & 2,2 \\
\hline Isidro Ayora & $22(0,3)$ & $14(0,2)$ & $4(0,9)$ & 0,3 & 0,6 & 1,2 & 0,2 & 0,3 & 0,1 \\
\hline Lomas de S. & $26(0,3)$ & $19(0,3)$ & $4(0,9)$ & 0,4 & 0,7 & 1,6 & 0,2 & 0,3 & 0,1 \\
\hline Milagro & $510(6,5)$ & $434(6,5)$ & $23(5,0)$ & 7,1 & 14,0 & 37,5 & 5,5 & 2,0 & 0,3 \\
\hline Naranjal & $217(2,7)$ & $177(2,7)$ & $45(9,8)$ & 3,0 & 6,0 & 15,3 & 2,2 & 3,9 & 0,6 \\
\hline Naranjito & $92(1,2)$ & $53(0,8)$ & $11(2,4)$ & 1,3 & 2,5 & 4,6 & 0,7 & 0,9 & 0,1 \\
\hline Nobol & $52(0,7)$ & $49(0,7)$ & $8(1,7)$ & 0,7 & 1,4 & 4,2 & 0,6 & 0,7 & 0,1 \\
\hline Palestina & $29(0,4)$ & $28(0,4)$ & $3(0,7)$ & 0,4 & 0,8 & 2,4 & 0,4 & 0,3 & 0,0 \\
\hline Pedro Carbo & $40(0,5)$ & $51(0,8)$ & $5(1,1)$ & 0,6 & 1,1 & 4,4 & 0,6 & 0,4 & 0,1 \\
\hline Playas & $91(1,2)$ & $76(1,1)$ & $3(0,7)$ & 1,3 & 2,5 & 6,6 & 1,0 & 0,3 & 0,0 \\
\hline Salitre & $56(0,7)$ & $56(0,8)$ & $11(2,4)$ & 0,8 & 1,5 & 4,8 & 0,7 & 0,9 & 0,1 \\
\hline Samborondón & $263(3,3)$ & $182(2,7)$ & $10(2,2)$ & 3,7 & 7,2 & 15,7 & 2,3 & 0,9 & 0,1 \\
\hline Santa Lucía & $60(0,8)$ & $67(1,0)$ & $9(2,0)$ & 0,8 & 1,6 & 5,8 & 0,8 & 0,8 & 0,1 \\
\hline Simón Bolívar & $46(0,6)$ & $42(0,6)$ & $1(0,2)$ & 0,6 & 1,3 & 3,6 & 0,5 & 0,1 & 0,0 \\
\hline Yaguachi & $215(2,7)$ & $184(2,8)$ & $24(5,2)$ & 3,0 & 5,9 & 15,9 & 2,3 & 2,1 & 0,3 \\
\hline LOS RÍOS & $1.036(8,4)$ & $829(8,6)$ & $135(15,0)$ & 13,7 & 133,1 & 67,0 & 80,0 & 10,9 & 13,0 \\
\hline Baba & $15(1,4)$ & $14(1,7)$ & $2(1,5)$ & 0,2 & 1,9 & 1,2 & 1,4 & 0,2 & 0,2 \\
\hline Babahoyo & $344(33,2)$ & $307(37,0)$ & $30(22,2)$ & 4,8 & 44,2 & 26,5 & 29,6 & 2,6 & 2,9 \\
\hline Urdaneta & $1(0,1)$ & - & $2(1,5)$ & 0,0 & 0,1 & - & - & 0,2 & 0,2 \\
\hline Mocache & $15(1,4)$ & $18(2,2)$ & $3(2,2)$ & 0,2 & 1,9 & 1,6 & 1,7 & 0,3 & 0,3 \\
\hline Montalvo & $32(3,1)$ & $25(3,0)$ & $8(5,9)$ & 0,4 & 4,1 & 2,2 & 2,4 & 0,7 & 0,8 \\
\hline Palenque & $7(0,7)$ & $1(0,1)$ & - & 0,1 & 0,9 & 0,1 & 0,1 & - & - \\
\hline Puebloviejo & $46(4,4)$ & $23(2,8)$ & $11(8,1)$ & 0,6 & 5,9 & 2,0 & 2,2 & 0,9 & 1,1 \\
\hline Quevedo & $216(20,8)$ & $155(18,7)$ & $22(16,3)$ & 3,0 & 27,8 & 13,4 & 15,0 & 1,9 & 2,1 \\
\hline Quinsaloma & $14(1,4)$ & $7(0,8)$ & $5(3,7)$ & 0,2 & 1,8 & 0,6 & 0,7 & 0,4 & 0,5 \\
\hline San Jacinto B.F. & $141(13,6)$ & $119(14,4)$ & $15(11,1)$ & 2,0 & 18,1 & 10,3 & 11,5 & 1,3 & 1,4 \\
\hline Valencia & $38(3,7)$ & $29(3,5)$ & $9(6,7)$ & 0,5 & 4,9 & 2,5 & 2,8 & 0,8 & 0,9 \\
\hline
\end{tabular}




\begin{tabular}{|c|c|c|c|c|c|c|c|c|c|}
\hline & & Lesionados & Fallecidos & Accident & & Lesi & & Letal & \\
\hline & $n(\% n)$ & $n(\% n)$ & n $(\% n)$ & TAAT $_{b}$ & TAAT $_{\text {a }}$ & TLSAT $_{b}$ & TLSAT $_{a}$ & TLEAT $_{b}$ & TLEAT $_{\mathrm{a}}$ \\
\hline Ventanas & $92(8,9)$ & $68(8,2)$ & $11(8,1)$ & 1,3 & 11,8 & 5,9 & 6,6 & 0,9 & 1,1 \\
\hline Vinces & $75(7,2)$ & $63(7,6)$ & $17(12,6)$ & 1,0 & 9,6 & 5,4 & 6,1 & 1,5 & 1,6 \\
\hline MANABÍ & $1.062(8,6)$ & $891(9,2)$ & $141(15,6)$ & 14,0 & 77,5 & 72,0 & 83,9 & 11,4 & 13,3 \\
\hline 24 de Mayo & $2(0,2)$ & $1(0,1)$ & $2(1,4)$ & 0,0 & 0,1 & 0,1 & 0,1 & 0,2 & 0,2 \\
\hline Bolívar & - & - & - & - & - & - & - & - & - \\
\hline Chone & $175(16,5)$ & $143(16,0)$ & $24(17,0)$ & 2,4 & 12,8 & 12,3 & 13,5 & 2,1 & 2,3 \\
\hline El Carmen & $26(2,4)$ & $42(4,7)$ & $17(12,1)$ & 0,4 & 1,9 & 3,6 & 4,0 & 1,5 & 1,6 \\
\hline Flavio Alfaro & $16(1,5)$ & $20(2,2)$ & $5(3,5)$ & 0,2 & 1,2 & 1,7 & 1,9 & 0,4 & 0,5 \\
\hline Jama & $4(0,4)$ & $1(0,1)$ & $1(0,7)$ & 0,1 & 0,3 & 0,1 & 0,1 & 0,1 & 0,1 \\
\hline Jaramijó & $8(0,8)$ & $12(1,3)$ & $3(2,1)$ & 0,1 & 0,6 & 1,0 & 1,1 & 0,3 & 0,3 \\
\hline Jipijapa & $133(12,5)$ & $175(19,6)$ & $10(7,1)$ & 1,8 & 9,7 & 15,1 & 16,5 & 0,9 & 0,9 \\
\hline Junín & - & - & - & - & - & - & - & - & 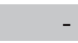 \\
\hline Manta & $333(31,4)$ & $174(19,5)$ & $17(12,1)$ & 4,6 & 24,3 & 15,0 & 16,4 & 1,5 & 1,6 \\
\hline Montecristi & $7(0,7)$ & $1(0,1)$ & $1(0,7)$ & 0,1 & 0,5 & 0,1 & 0,1 & 0,1 & 0,1 \\
\hline Olmedo & $1(0,1)$ & $3(0,3)$ & - & 0,0 & 0,1 & 0,3 & 0,3 & 0,0 & 0,0 \\
\hline Paján & $27(2,5)$ & $19(2,1)$ & $3(2,1)$ & 0,4 & 2,0 & 1,6 & 1,8 & 0,3 & 0,3 \\
\hline Pedernales & $11(1,0)$ & $21(2,4)$ & $6(4,3)$ & 0,2 & 0,8 & 1,8 & 2,0 & 0,5 & 0,6 \\
\hline Pichincha & $5(0,5)$ & $18(2,0)$ & $7(5,0)$ & 0,1 & 0,4 & 1,6 & 1,7 & 0,6 & 0,7 \\
\hline Portoviejo & $243(22,9)$ & $214(24,0)$ & $23(16,3)$ & 3,4 & 17,7 & 18,5 & 20,2 & 2,0 & 2,2 \\
\hline Puerto López & - & - & - & - & - & - & - & - & 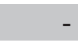 \\
\hline Rocafuerte & $20(1,9)$ & $21(2,4)$ & $5(3,5)$ & 0,3 & 1,5 & 1,8 & 2,0 & 0,4 & 0,5 \\
\hline San Vicente & $23(2,2)$ & $8(0,9)$ & $7(5,0)$ & 0,3 & 1,7 & 0,7 & 0,8 & 0,6 & 0,7 \\
\hline Santa Ana & $5(0,5)$ & $4(0,4)$ & - & 0,1 & 0,4 & 0,3 & 0,4 & 0,0 & 0,0 \\
\hline Sucre & $17(1,6)$ & $10(1,1)$ & $5(3,5)$ & 0,2 & 1,2 & 0,9 & 0,9 & 0,4 & 0,5 \\
\hline Tosagua & $6(0,6)$ & $4(0,4)$ & $5(3,5)$ & 0,1 & 0,4 & 0,3 & 0,4 & 0,4 & 0,5 \\
\hline SANTA ELENA & $464(3,7)$ & $411(4,3)$ & $43(4,8)$ & 6,1 & 150,3 & 33,2 & 88,6 & $3,5^{*}$ & 9,3 \\
\hline La Libertad & $151(32,5)$ & $134(32,6)$ & $7(16,3)$ & 2,1 & 48,9 & 11,6 & 28,9 & 0,6 & 1,5 \\
\hline Salinas & $107(23,1)$ & $88(21,4)$ & $6(14,0)$ & 1,5 & 34,7 & 7,6 & 19,0 & 0,5 & 1,3 \\
\hline Santa Elena & $206(44,4)$ & $189(46,0)$ & $30(69,8)$ & 2,9 & 66,7 & 16,3 & 40,7 & 2,6 & 6,5 \\
\hline SANTO DOMINGO T. & $792(6,4)$ & $688(7,1)$ & $68(7,5)$ & 10,5 & 215,2 & 55,6 & 86,9 & 5,5 & 8,6 \\
\hline
\end{tabular}

TAATb = Tasa de Accidentabilidad Bruta por Accidentes de Tránsito (por cada 100.000 habitantes). TAATa = Tasa de Accidentabilidad Ajustada por Accidentes de Tránsito (por cada 100.000 habitantes). TLSATb = Tasa de Lesividad Bruta por Accidentes de Tránsito (por cada 1.000 siniestros). TLSATa = Tasa de Lesividad Ajustada por Accidentes de Tránsito (por cada 100 siniestros). TLEATb = Tasa de Letalidad Bruta por Accidentes de Tránsito (por cada 1.000 siniestros). TLEATa = Tasa de Letalidad Ajustada por Accidentes de Tránsito (por cada 100 siniestros). Coeficiente de Correlación de Pearson $\left({ }^{*} p<0,05 ;{ }^{* \star} p<0,01 ;{ }^{* *} p<0,001\right)$.

Guayas con 37,0 fallecidos por cada 1.000 sinies- $^{-}$ tros, Manabí con 11,4 fallecidos por cada 1.000 siniestros y con 10,9 fallecidos por cada 1.000 siniestros la provincia de Los Ríos, Tabla 3.

Se observa que la mayor tasa de lesividad bruta (535,8 x1.000 siniestros) y accidentabilidad (104,5 x100.000 habitantes) se encuentra en la provincia del Guayas, con valores inferiores, Manabí (72,0 x1.000 siniestros; 14,0 x100.000 habitantes) y Los Ríos (67,0 x1.000 siniestros; $13,7 \times 100.000$ habitantes).

Por cantones provinciales, Santo Domingo de los Tsachilas $(8,6)$, Santa Elena $(6,5)$, Quinindé $(4,4)$, Machala $(3,4)$, Chone $(2,3)$, Guayaquil $(2,2)$, Portoviejo $(2,2)$ y Quevedo $(2,1)$ representan las mayores tasas de letalidad ajustada por AT en la región y según cantones y tasas de lesividad ajustadas, Guayaquil $(52,6)$, Quinindé $(44,4)$, Esmeraldas (41,3), Santa Elena 40,7), Babahoyo (29,6), La Libertad $(28,9)$, Machala $(24,9)$ y Portoviejo $(20,2)$.

Finalmente, Guayaquil (131,9 x 100.000 habitantes), Santa Elena (66,7 x 100.0000 ha- bitantes), Machala (53,1 x 100.000 habitantes), La Libertad (48,9 x 100.0000 habitantes) y Babahoyo (44,2 x 100.000 habitantes) representan las mayores tasas ajustadas de accidentabilidad por cantón provincial.

Se observa una asociación estadísticamente significativa entre la tasa de lesividad y el número de habitantes de los cantones provinciales del Guayas $(<0,001)$, igulamente, entre la tasa de letalidad y el número de habitantes de los cantones provinciales de El Oro $(\mathrm{p}<0,05)$, Esmeraldas y Guayas $(\mathrm{p}<0,01)$. No encontrandose asociación estadísticamentes significativa para el resto de cantones provinciales de la Región Costa del Pacífico del país.

\section{DISCUSIÓN}

Nuestros resultados son similares a otros estudios realizados en países de Latinoamérica y El Caribe. En Perú, los siniestros por AT se originan principalmente en aquellas regiones con mayor densidad poblacional (Lima: 63,8\%) y las mayores tasas de letalidad y mortalidad 
se concentran en la región amazónica del país, ésta última debido a la inaccesibilidad de atención pre-hospitalaria a las víctimas afectadas (Choquehuanca, 2010).

Igualmente, en otro estudio realizado en Costa Rica, ubican el $61 \%$ de los siniestros y el $70 \%$ de lesionados por AT en carreteras cantonales interurbanas (Guápiles y Cariari), principalmente por colisión entre vehículos y por causas relacionadas con el estado de la vía (Castro, 2011; Agüero, 2013).

Del análisis de egresos hospitalarios por AT de la Red Pública del Departamento de Risaralda (Colombia), sitúa a este departamento como el primero en tasa de letalidad (16 x100.000 habitantes) a nivel nacional (media 14 x100.000 habitantes) (Martínez, Muñoz \& Escobar, 2004), valores inferiores a las provincias de Ecuador; Morona Santiago (24 x100.000 habitantes), Cañar (21 x100.000 habitantes), Sucumbíos Imbabura (20 x100.000 habitantes), Bolívar (18 x100.000 habitantes) y Los Ríos - Chimborazo (17 x100.000 habitantes).

Esta investigación no está exenta de limitaciones y se debe tener precaución al interpretar sus resultados. En primer lugar, al desconocer el punto kilométrico de la red vial dónde ocurrió el AT impide precisar con detalle su localización geográfica (puntos negros-críticos) (Brubacher, et al., 2016). En segundo lugar, al tratarse de un estudio transversal, imposibilita conocer la evolución y tendencia de los AT según regiones, provincias y cantones del país. Finalmente, en tercer lugar, las estadísticas oficiales recopiladas por la Agencia Nacional de Tránsito dificultan la combinación geográfica de los eventos por AT con otras variables (temporales, vehículo y usuario implicado, causas y tipología del accidente) y de este modo comprender con mayor exactitud esta problemática en el país (Leveau \& Ubeda, 2012).

CONCLUSIONES

En conclusión y a pesar de las limitaciones descritas anteriormente, el análisis de este estudio proporciona una aproximación para la identificación a nivel nacional de aquellas provincias y cantones con mayores tasas de accidentabilidad, lesividad y letalidad por AT, aportando evidencias para el diseño de políticas públicas y el establecimiento de programas en seguridad vial en ciertas zonas geográficas con mayores tasas.

De los hallazgos encontrados, se ha observado la asociación positiva entre la población y las tasas de lesivividad y letalidad por AT en los cantones provinciales, este hecho puede ser debido a la densidad del parque automotriz y su correspondiente flujo vehicular (desplazamiento - movilidad de la población), así como, a la posible precariedad de la infraestructura vial (carreteras rurales, interurbanas y urbanas) para conductores y peatones (Batrakova \& Gredasova, 2016). No obstante, las causas que originan los AT se deben a la combinación multifactorial de variables (Akanbi, Owaba, \& Oluleye, 2009), por lo que no podemos atribuirlos considerando solamente la zona geográfica.

Se revela la necesidad de mejorar los sistemas de información estadística de los AT por parte los organismos públicos del país (WHO, 2015; Gómez García, et al., 2016) y la aplicación otros métodos de análisis que permitan el desarrollo de investigaciones más precisas (Hernández Vásquez, et al., 2016), así como, considerar igualmente las características sociales y económicas.

Aunque en Ecuador, en los últimos años se observa una tendencia a la disminución de la accidentabilidad y letalidad por AT, sigue siendo un problema de salud pública (Algora Buenafé, et al., 2017b). Se requiere un mayor esfuerzo para optimizar las actividades destinadas al cumplimiento de las normas legales de tránsito y educación vial para la población (Algora Buenafé, et al., 2017a).

\section{REFERENCIAS BIBLIOGRÁFICAS}

Agencia Nacional de Tránsito de Ecuador. (2008). Ley Orgánica de Transporte Terrestre, Tránsito y Seguridad Vial. Ecuador: ANT. Registro Oficial Suplemento N. ${ }^{\circ}$ 398, 7 de agosto del 2008.

Agencia Nacional de Tránsito de Ecuador. (2011). Ley Orgánica de Transporte Terrestre, Tránsito y Seguridad Vial. Ecuador: ANT. Registro Oficial Suplemento N. ${ }^{\circ}$ 415, 29 de marzo del 2011.

Agencia Nacional de Tránsito. (2016). Estadísticas de transporte terrestre y seguridad vial. Ecuador: ANT. Recuperado de http://www. ant.gob.ec/index.php/noticias/estadisticas\#. V4IGYFcWUvP. Consultado el 17-02-2018.

Agüero Valverde, J. (2013). Multivariate spatial models of excess crash frequency at area level: case of Costa Rica. Accident Analysis \& Prevention, 59: 365-373. doi: 10.1016/j. aap.2013.06.014

Akanbi, O.G., Owaba, C., Oluleye, A.E. (2009). Human factors in traffic accidents in Lagos, Nigeria. An International Journal, 18(4): 397409. doi: 10.1108/09653560910984456 
Algora Buenafé, A.F., Russo Puga, M., Suasnavas Bermúdez, P.R., Merino Salazar, P., Gómez García, A.R. (2017b). Tendencias de los accidentes de tránsito en Ecuador: 2000-2015. Revista Gerencia y Políticas de Salud, 16(33): 52-58. doi: 10.11144/Javeriana.rgps16-33.tate

Algora Buenafé, A.F., Suasnavas Bermúdez, P.R., Merino Salazar, P., Gómez García, A.R. (2017a). Epidemiological study of fatal road traffic accidents in Ecuador. Australasian Medical Journal, 10(3), 238-245. doi: 10.21767/ AMJ.2017.2951

Algora Buenafé, A.F., Tapia Claudio, O.M., Gómez García, A.R. (2017c). Análisis espacial de los accidentes de tránsito en los Cantones de la Provincia de Pichincha, 2016. CienciAmérica, 6 (1): 24-30.

Ameratunga, S., Hijar, M., Norton, R. (2006). Road-traffic injuries: confronting disparities toaddress a global-health problem. Lancet, 367, 1533-1540. doi: 10.1016/S0140-6736(06)68654-6

Bastos, J,T, Shen, Y., Hermans, E., Brijs, T., Wets, G., Ferraz, A.C. (2015). Traffic fatality indicators in Brazil: State diagnosis based on data envelopment analysis research. Accident Analysis \& Prevention, 81: 61-73. doi: 10.1016/j. aap.2015.01.024

Batrakova, A., Gredasova, O. (2016). Influence of Road Conditions on Traffic Safety. Procedia Engineering, 134: 196-204. doi: 10.1016/j. proeng.2016.01.060

Brubacher, J.R., Chan, H., Erdelyi, S., Schuurman, N., Amram, O. (2016). The Association between Regional Environmental Factors and Road Trauma Rates: A Geospatial Analysis of 10 Years of Road Traffic Crashes in British Columbia, Canada. PLoS ONE 11(4): e0153742. doi:10.1371/journal. pone.0153742

Castro Delgado, F. (2011). Análisis de la distribución espacial de los accidentes de tránsito. Revista Geográfica de América Central, 2011; 2: 1-43.

Cheng, W., Washington, S.P. (2005). Experimental evaluation of hotspot identification methods. Accident Analysis \& Prevention, 37(5): 870-881. doi: 10.1016/j.aap.2005.04.015

Choquehuanca Vilca, V., Cárdenas García, F., Collazos Carhuay, J., Mendoza Valladolid, W. (2010). Perfil epidemiológico de los accidentes de tránsito en el Perú, 2005-2009. Revista Peruana de Medicina Experimental y Salud Pública, 27(2): 162-169.

Fuentes, C.M., Hernández, V. (2009). La estructura espacial urbana y la incidencia de accidentes de tránsito en Tijuana, Baja California
(2003-2004). Frontera Norte, 21(42): 109-138. doi: 10.17428/rfn.v21i42.966

Galarza Velastegui, L.A., Merino Salazar, P., Algora Buenafé, A.F., Gómez García, A.R. (2017). Estudio geoespacial de los accidentes de tránsito en la Región Amazónica Ecuatoriana. CienciAmérica, 6 (2): 21-26.

Gómez García, A.R., Chérrez Miño, M.C., Russo Puga, M., González Jijón, L.A., Suasnavas Bermúdez, P.R., Celín Ortega, F.A. (2016). Caracterización de la mortalidad por accidentes de tránsito en Ecuador, 2015. CienciAmérica, 5: 22-31.

Hernández Hernández V. (2012). Análisis exploratorio espacial de los accidentes de tránsito en Ciudad Juárez, México. Revista Panamericana de Salud Pública, 31(5):396-402.

Hernández Vásquez, A., Azañedo, D., Bendezú Quispe, G., Pacheco Mendoza, J., Chaparro, R.M. (2016). Sistemas de información geográfica: aplicación práctica para el estudio de atropellos en el Cercado de Lima, Perú. Revista Peruana de Medicina Experimental y Salud Píblica, 33(4): 725-731. doi: 10.17843/ rpmesp.2016.334.2558

Híjar M., Pérez Núñez, R., Inclán Valadez, C., Silveira Rodrigues, E.M. (2012). Road safety legislation in the Americas. Revista Panamericana de Salud Pública, 32(1): 70-76. doi: 10.1590/S1020-49892012000700011

Instituto Nacional de Estadística y Censos. (2010). Población y Demografía. Ecuador: INEC. Recuperado de http://www.ecuadorencifras.gob. ec/censo-de-poblacion-y-vivienda. Consultado el 9-02-2018.

Kumar, G.A., Dilip, T.R., Dandona, L., Dandona, R. (2012). Burden of out-of-pocket expenditure for road traffic injuries in urban India. BMC Health Services Research, 12: 285. doi: 10.1186/1472-6963-12-285

Leveau, C.M., Ubeda, C. (2012). Muertes por lesiones de tránsito en Argentina: un análisis espacial para el Período 2001-2009. Revista Panamericana de Salud Pública, 31(5): 439-442. Martínez, J.W., Muñoz, L.P., Escobar, M.V. (2004). Lesiones por accidentes de tránsito en Risaralda. Investigaciones Andina, 9: 14-20.

Mayou, R., Bryant, B. (2003). Consequences of road traffic accidents for different types of road user. Injury, 34, 197-202. doi: 10.1016/ S0020-1383(02)00285-1

McWade, C.M., McWade, M.A., Quistberg, DA, McNaughton, C.D., Wang, L., Bux, Z., Forget, N.P. (2017). Epidemiology and mapping of serious and fatal road traffic injuries in Guyana: 
results from a cross-sectional study. Injury Prevention, 23(5): 303-308. doi: 10.1136/injuryprev-2016-042119

Montella, A. (2010). A comparative analysis of hotspot identification methods. Accident Analysis \& Prevention, 42: 571-581. doi: 10.1016/j.aap.2009.09.025

Pan American Health Organization. (2013). Status Report on Road Safety in Americas Region. Washington, DC: PAHO. Recuperado de http://www.paho.org/hq/ index.php?option $=$ com_docman\&task $=$ doc view\&gid $=20941$ \& Itemid $=2 \% 2070$ \&lang $=e n$. Consultado el 19-012-2017.

Qin, E.S., Jennissen, C.A., Wadman, C.A., Denning, G.M. (2017). Using Geospatial Mapping to Determine the Impact of All-Terrain Vehicle Crashes on Both Rural and Urban Communities. The Western Journal of Emergency Medicine, 18(5): 913-922. doi: 10.5811/ westjem.2017.6.34404

Restrepo Morales, J,A., Medina Hurtado, S., Vallejo Mesa, J. (2016). Pérdidas económicas para las compañías aseguradoras derivadas de personas lesionadas en accidentes de tránsito: Aplicación de un modelo de pérdidas agregadas. Revista Gerencia y Políticas de Salud, 15(30): 80-93. doi: 10.11144/Javeriana. rgyps15-30.peca
Rissanen, R,. Berg, H.Y,, Hasselberg, M. (2017). Quality of life following road traffic injury: A systematic literature review. Accident; Analysis and Prevention, 108: 308-320. doi: 10.1016/j.aap.2017.09.013

Staton, C., Vissoci, J., Gong, E., Toomey, N., Wafula, R., Abdelgadir, J., et al. (2016). Road Traffic Injury Prevention Initiatives: A Systematic Review and Metasummary of Effectiveness in Low and Middle Income Countries. PLoS One, 11(1): e0144971. doi: 10.1371/journal. pone. 0144971

World Health Organization. (2007). Ten statistical highlights in global public health: Part 1. Geneva: WHO. Recuperado de http://www.who. int/gho/publications/world_health_statistics/ whostat2007_10highlights.pdf. Consultado el 06-09-2017.

World Health Organization. (2015). Global status report on road safety 2015. Geneva: WHO. Recuperado de http://apps.who.int/iris/bitstr eam/10665/189242/1/9789241565066_eng. pdf?ua=1. Consultado el 02-011-2017. 